\title{
Diazoxide Attenuates Ischemic Myocardial Injury in a Porcine Model
}

\author{
Henna Sarja, MB,${ }^{1}$ Tuomas Anttila, MD,${ }^{1}$ Caius Mustonen, MB,${ }^{1}$ Hannu-Pekka Honkanen, MB, ${ }^{1}$ \\ Johanna Herajärvi, MD ${ }^{1}$ Henri Haapanen, $\mathrm{MD},{ }^{1}$ Hannu Tuominen, $\mathrm{MD}, \mathrm{PhD},{ }^{2}$ Ilkka Miinalainen, $\mathrm{PhD},{ }^{3}$ \\ Tatu Juvonen, MD, PhD, ${ }^{1,4}$ Vesa Anttila, MD, $\mathrm{PhD}^{1,5}$
}

\begin{abstract}
${ }^{1}$ Research Unit of Surgery, Anesthesia and Intensive Care, University of Oulu and Medical Research Center, Oulu; ${ }^{2}$ Department of Pathology, Medical Research Center Oulu, Oulu University Hospital and University of Oulu; ${ }^{3}$ Medical Research Center Oulu, Research Unit of Biomedicine, University of Oulu and University Hospital Oulu, Oulu, Finland; ${ }^{4}$ Department of Cardiac Surgery, Heart and Lung Center, Helsinki University Central Hospital, Helsinki University Hospital, Helsinki, Finland; ${ }^{5}$ Heart Center, University of Turku and Turku University Hospital, Turku, Finland
\end{abstract}

\section{ABSTRACT}

Background: We hypothesized that diazoxide, a mitochondrial ATP-sensitive potassium channel opener, has cardioprotective effects during acute myocardial ischemia. Diazoxide is suggested to act through protein kinase $\mathrm{C} \varepsilon$ (PKCE) activation.

Methods: Twelve piglets were randomly assigned to receive intravenous infusion of diazoxide $(3.5 \mathrm{mg} / \mathrm{kg})$ with solvent or only solvent (6 animals per group) before cardiac ischemia. Myocardial ischemia was induced by occluding the left circumflex artery (LCX) for 40 minutes. The reperfusion and follow-up period lasted for three hours. Throughout the experiment hemodynamic measurements and blood samples were collected, and after the follow-up period the hearts were harvested for transmission electron microscopy (TEM) as well as histopathological and immunohistochemical analyses.

Results: TEM showed less ischemic damage on a cellular level in the diazoxide group $(P=.004)$ than in the control group. Creatinine kinase MB levels $\left(P \mathrm{t}^{*} \mathrm{~g}=.030\right)$ were lower, and oxygen consumption $\left(P \mathrm{t}^{*} \mathrm{~g}=.037\right)$ and delivery $(P g=.038)$ were higher in the diazoxide group compared to the controls.

Conclusion: Diazoxide preserves myocardial cellular structure and cellular function, and thus it may have benefits in treating ischemic myocardial injury.

\section{INTRODUCTION}

Ischemic cardiac injury is one of the leading causes of mortality and morbidity worldwide and extensive amount of research on its treatment has been performed. In 1997, Garlid et al directed attention to the cardioprotective effect of the mitochondrial ATP-sensitive potassium channel (mitoKATP) opener diazoxide [Garlid 1997]. Pharmacological preconditioning $(\mathrm{PPC})$ by diazoxide can mimic the cardioprotective

Received March 14, 2017; received in revised form fune 12, 2017; accepted fune 14, 2017.

Correspondence: Henna Sarja, MB, Research Unit of Surgery, Anesthesia and Intensive Care, University of Oulu and Medical Research Center, PO Box 21, 90029 OYS, Oulu, Finland; +358-407606591 (e-mail: henna.sarja@student.oulu.fi). effect of ischemic preconditioning (IPC) [Baines 1999; Ghosh 2000; Sanada 2001], which is one of the most potent mechanisms for reducing ischemic injury. IPC is produced by exposing the cardiac tissue to brief ischemic episodes before subsequent prolonged ischemia [Murry 1986] and it can be attenuated by a mitoK $\mathrm{ATP}_{\text {}}$ channel blocker, indicating that mitoKATP might be the downstream target of IPC [Fryer 2000; Fryer 2001]. However, the mechanism of preconditioning is not fully understood.

Experimental models using diazoxide for myocardial ischemic preconditioning have shown that the infarct area is reduced [Baines 1999; Sanada 2001] and myocardial swelling secondary to stress is alleviated [Mizutani 2006; Sellito 2010]. Additionally, in clinical settings diazoxide added to cardioplegia has prevented ischemia and reperfusion-induced mitochondrial swelling [Deja 2009]. Furthermore, it has been suggested that in the failing heart, mitochondrial dysfunction induces apoptosis, interstitial fibrosis and myocyte hypertrophy [Tsutsui 2006]. Therefore, preservation of mitochondrial

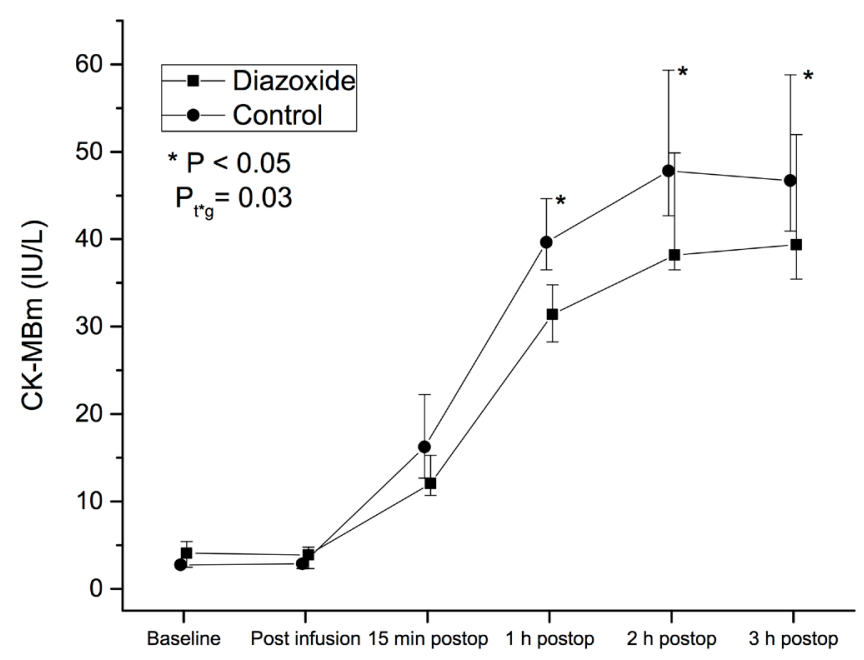

Figure 1. Creatine kinase isoenzyme MB (CK-MBm). The CK-MBm release was significantly higher in the control group over time. In different time points starting from one hour after the LCX occlusion until the end of the experiment, CK-MBm release was significantly higher in the control group. 
Table 1. Immunohistochemical Methods Used in This Study

\begin{tabular}{lccccc}
\hline Antibody (clone) & Dilution & Retrieval & Detection Kit & Incubation Time & Manufacturer \\
\hline Nrf2 (ab 62352) & $1: 200$ & Tris-EDTA & EnVision Dako, kit K5007 & 90 min & Abcam \\
OGG1 (NB100-106) & $1: 1000$ & Citrate buffer & EnVision Dako, kit K5007 & Overnight & Novus Biologicals \\
8-OHdG (N45.1) & $1: 75$ & Citrate buffer & Life technologies, Histostain Plus, Broad spectrum & 60 min at $42^{\circ} \mathrm{C}$ & Japan Institute for the Control of Aging \\
PKCE (orb158162) & $1: 500$ & Tris-EDTA & EnVision Dako, kit K5007 & Overnight & Biorbyt
\end{tabular}

function may be a promising therapeutic target for preventing ischemic injury in the myocardium.

One of the cardioprotective mechanisms of mitoK ${ }_{\text {ATP }}$ opening is suggested to be protein kinase $\mathrm{C} \varepsilon(\mathrm{PKC} \varepsilon)$ activation [Garlid 2013; Li 2014]. In myocytes, PKC 2 targets various mitochondrial proteins such as the mitochondrial permeability transition pore (mPTP) by inhibiting its opening [Baines 2003]. The opening of the mPTP has been demonstrated in reperfusion injury [Griffiths 1995], and due to the pore opening, mitochondrial swelling and a release of apoptogenic proteins are induced [Yang 1998].

In the present study, the effect of diazoxide on the oxidative stress in the myocardium was assessed by measuring the activation of 8-hydroxydeoxyguanosine (8-OHdG), which is produced when the DNA is under oxidative stress [Valavanidis 2009]. 8-Oxoguanine glycosylase (OGG1) then removes 8-OHdG from the damaged DNA [de Souza-Pinto 2001]. Furthermore, nuclear erythroid-related factor-2 (Nrf2) indicates oxidative stress by activating the antioxidant response element in the nucleus, which then initiates the cell protection by the transcription of various cytoprotective proteins [Itoh 1997; Itoh 1999].

Much uncertainty still exists about the relationship between the mitoKATP channels and cardioprotection. The aim of the study was to test the hypothesis that mitoKATP channel opener diazoxide improves cardiac protection against ischemic insult by preserving mitochondrial function. In addition, we intended to investigate whether the pharmacological preconditioning by diazoxide is an efficient treatment of ischemic myocardial injury.

\section{MATERIAL AND METHODS}

All the animals used in this study received human care in compliance with the Principles of Laboratory Animal Care formulated by the National Society for Medical Research and the Guide for the Care and Use of Laboratory Animals prepared by the Institute of Laboratory Animal Resources, National Research Council, The National Academies Press, 1996. The study was approved by the Research Animal Care and Use Committee of University of Oulu.

\section{Experimental Setting}

Twelve female piglets $(19-26 \mathrm{~kg})$ from a native stock were randomized into two groups: the diazoxide group $(n=6)$ and the control group $(n=6)$. Both groups underwent a 40-minute occlusion of left circumflex artery (LCX). Prior to the LCX occlusion the diazoxide group was administered an intravenous infusion of diazoxide $(3.5 \mathrm{mg} / \mathrm{kg}+10 \mathrm{~mL}$ $\mathrm{NaOH}+40 \mathrm{~mL} \mathrm{NaCl})$. The control piglets received a $50 \mathrm{~mL}$ infusion $(10 \mathrm{~mL} \mathrm{NaOH}+40 \mathrm{~mL} \mathrm{NaCl})$ without diazoxide.

\section{Anesthesia Protocol}

The animals were sedated with an intramuscular injection of esketamine $(250 \mathrm{mg})$, midazolam $(40 \mathrm{mg})$ and medetomidine $(2 \mathrm{mg})$. For fluid and drug administration, peripheral cannulations into auricular veins were performed. Anesthesia was inducted with fentanyl $(0.5 \mathrm{mg})$. The animals were then intubated with a cuffed $6.0 \mathrm{~mm}$ endotracheal tube and ventilated by a respirator with a $55 \% / 45 \%$ of oxygen/air-mixture. Throughout the experiment the anesthesia was maintained by a continuous infusion of rocuronium $(1.5 \mathrm{mg} / \mathrm{kg} / \mathrm{h})$, midazolam $(0.25 \mathrm{mg} / \mathrm{kg} / \mathrm{h})$ and fentanyl $(0.015 \mathrm{mg} / \mathrm{kg} / \mathrm{h})$, as well as by an inhalation anesthesia of $1.0 \%$ sevoflurane. Preoperatively, cefuroxime $(1.5 \mathrm{~g})$ prophylaxis was administered. To exclude the possible beneficial effect of diazoxide-induced vasodilation, inotropes were used after the diazoxide infusion, if $>20 \%$ reduction of baseline mean arterial pressure (MAP) was detected.

\section{Hemodynamic Monitoring}

For blood sampling and hemodynamic monitoring, a pulmonary artery thermodilution catheter (Criticath $7.5 \mathrm{Fr}$, Argon, The Hague, Netherlands) was placed through the right femoral vein. An arterial line for monitoring invasive arterial pressure and blood sampling was inserted into the right femoral artery. Continuous electrocardiogram (ECG) was recorded throughout the experiment. Urine output was measured by inserting an $8 \mathrm{Ch}$ urinary catheter into the urinary bladder.

\section{Diazoxide Infusion}

Diazoxide (D9035, Sigma-Aldrich, St. Louis, MO, USA) was dissolved in $10 \mathrm{~mL}$ of $1 \mathrm{M}$ sodium hydroxide solution, which was further diluted in $40 \mathrm{~mL} \mathrm{NaCl}$. The infusion $(2.5 \mathrm{~mL} / \mathrm{min})$ was administered for 20 minutes through the pulmonary artery catheter after the baseline measurements and sampling. Following the infusion the catheter was rinsed with $10 \mathrm{~mL}$ of sodium chloride. The infusion was finished 20 minutes before the LCX occlusion.

\section{Myocardial Infarction}

A left-sided thoracotomy was performed in the fourth intercostal space to expose the bifurcation of the left anterior 


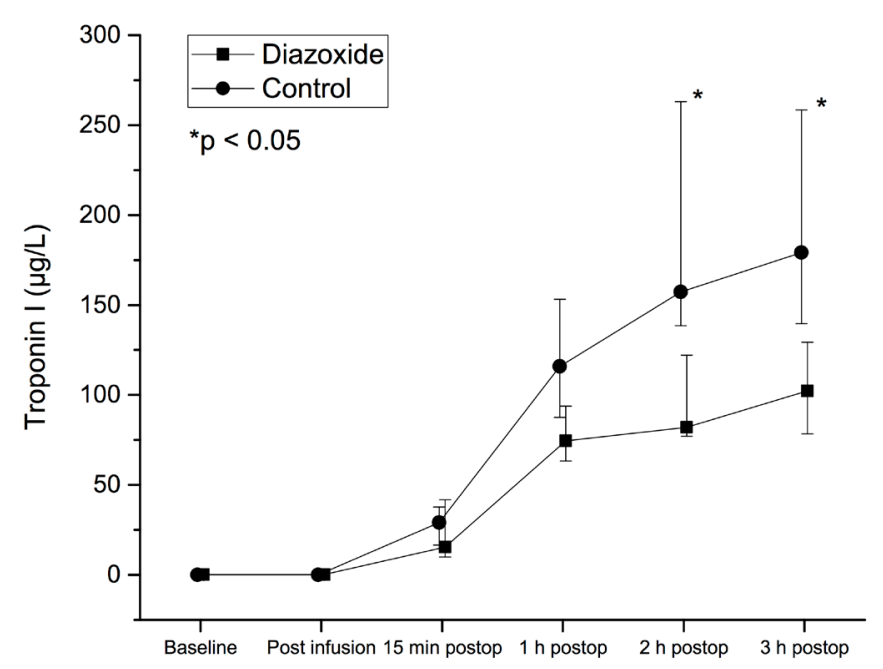

Figure 2. Troponin I. At two and three hours after the LCX occlusion, troponin I release was significantly higher in the control group.

descending (LAD) and the LCX coronary arteries. The LCX was occluded close to bifurcation using a silicone vascular loop for 40 minutes. ECG was used to indicate ST segment elevation. Reperfusion period was initiated after the 40-minute occlusion.

\section{Postoperative Management}

At the onset of reperfusion all the animals received topical papaverine hydrochloride $(30 \mathrm{mg}$ ) on the LCX coronary artery for preventing vasospasm. An additional dose of norepinephrine $(0.1 \mathrm{mg})$ was administered if necessary. After stable reperfusion was achieved, the thoracotomy incision was closed. After a 3-hour follow-up period the thoracic cavity was reopened. Myocardial samples for transmission electron microscopy (TEM) were taken from the infarcted area of each animal. Tissue samples were immersed in TEM fixative (1\% glutaraldehyde $4 \%$ formaldehyde mixture in $0.1 \mathrm{M}$ phosphate buffer, $\mathrm{pH}$ 7.4). Having obtained the sample, animals were euthanized by an intravenous injection of pentobarbital $(60 \mathrm{mg} / \mathrm{kg})$. Lastly, the heart was collected and immersed in $10 \%$ neutral formalin.

\section{Biochemical Data}

Arterial blood gas values, pH, electrolytes, plasma ionized calcium, glucose, hematocrit, hemoglobin, and lactate levels (i-STAT analyser, i-STAT, East Windsor, NJ, USA) were measured at baseline, 5 minutes after the diazoxide infusion, 15 minutes, 1 hour, 2 hours, and 3 hours after the LCX reperfusion. Simultaneously, serum troponin I and creatine kinase isoenzyme $\mathrm{MB}$ and full blood cell counts were also determined.

\section{Transmission Electron Microscopy}

Morphological structure of 10 cardiac samples (5 from both groups) was analyzed by using the transmission electron microscopy in a double-blinded manner. The fixed myocardial

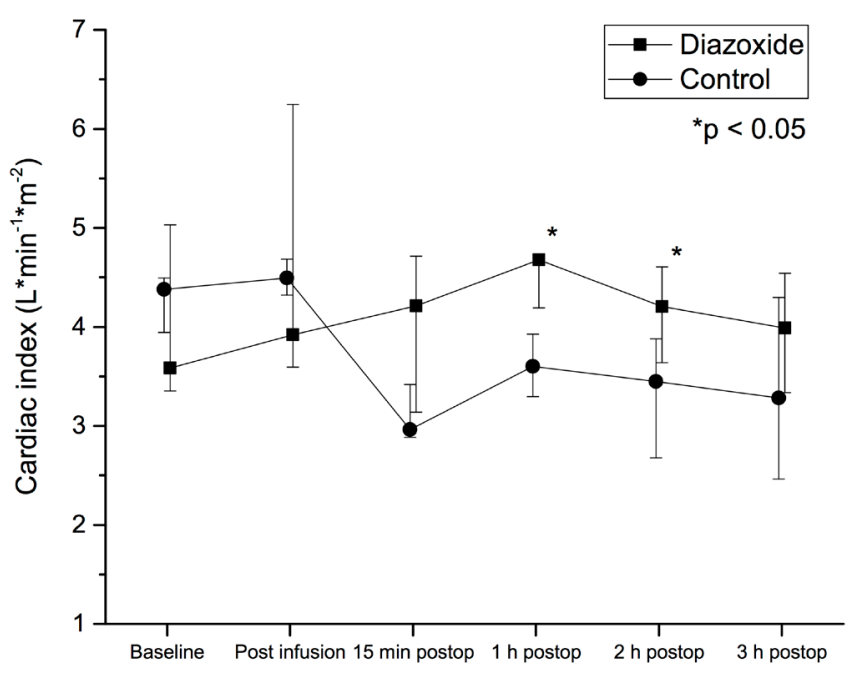

Figure 3. Cardiac index. After the LCX occlusion, the cardiac index was better preserved in the diazoxide group showing statistically significant differences at one and two hours postoperatively.

samples were postfixed in 1\% osmiumtetroxide, dehydrated in acetone and embedded in Epon LX 112 (Ladd Research Industries, Williston, Vermont, USA). Thin sections (70 nm) were cut with Leica Ultracut UCT ultramicrotome, stained in uranyl acetate and lead citrate and examined in Tecnai G2 Spirit transmission electron microscope (FEI Europe, Eindhoven, Netherlands). Images were captured by Quemesa CCD camera and analyzed using iTEM software (Olympus Soft Imaging Solutions GMBH, Munster, Germany). The total score of the damage to the myocardial ultrastructure was based on a semiquantitative analysis of the sum of the mitochondria $(0=$ normal, $1=$ swollen, 2 = amorphous matrix densities (AMD), disorganized cristae) and cardiomyocytes ( 0 = edema, 1 = vacuoles, loss of normal architecture, disintegrating sarcolemma, 2 = peripherally aggregated nuclear chromatin, lysis).

\section{Histopatbological Analysis and Immunobistochemistry}

The collected heart in 10\% neutral formalin was prepared for hematoxylin-eosin and immunohistochemical staining as described in our previous study [Jensen 2011]. Immunohistochemical staining method was conducted as described in Table 1. A total of six samples were collected from different regions of each harvested heart (infarct area, left anterior ventricle, left posterior ventricle, septum, left atrium). Two samples were obtained from infarct area, which were scored separately and then combined by maximum value to allow wider analysis of myocardial infarction.

Hematoxylin-eosin scoring was based on eosinophilia $(0-1)$, capillary congestion (0-1) and granulocyte infiltration $(0-1)$. The total regional score (0 to 3 ) was calculated by summing the aforementioned scores. The total histopathological score of the heart was the sum of all the regional scores (infarct area, left anterior ventricle, left posterior ventricle, septum, left atrium). The immunohistochemical analysis of 8-OHdG nuclear staining and Nrf2 cytoplasmic staining 


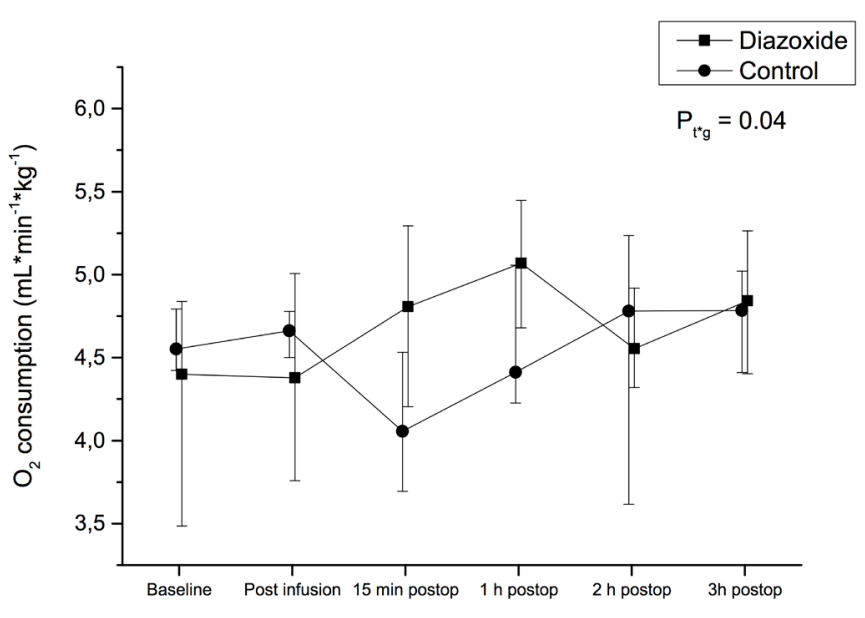

Figure 4. Oxygen consumption. In over time comparisons, oxygen consumption differed significantly between the groups. The most apparent difference was 15 minutes after the onset of reperfusion showing that the oxygen consumption was higher in the diazoxide group.

were based on semiquantitative analysis of the stained area ( 0 = negative, $1=$ under $50 \%$ of the section is positive, $2=50 \%$ of the section is positive, $3=$ over $50 \%$ of the section is positive). The immunohistochemical scores of OGG1 and PKCE cytoplasmic stainings in each specific sample were assessed by the intensity of the staining $(0=$ no staining, $1=$ weak staining, 2 = moderate staining, 3 = strong staining). An experienced pathologist scored the tissue samples by a blinded fashion.

\section{Statistical Analysis}

Statistical analyses were performed using SPSS (SPSS, version 23.0; SPSS, Chicago, IL) and SAS (version 9.4; SAS Institute, Cary, NC) statistical software packages. Continuous and ordinal variables are expressed as the median with 25 th -75 th percentiles or the mean with standard deviation. Complete independence was assumed across the animals (by random statement). The repeatedly measured data was analyzed using a Linear Mixed Model (LMM) with animals fitted as random, and the covariance pattern was chosen according to Akaike's information criteria. The $P$ values reported with LMM are as follows: $P$ between groups $(P g)$ indicates a level of difference between the groups; $P$ for time by group $\left(P \mathrm{t}^{*} \mathrm{~g}\right)$ indicates behavior between the groups over time. To explore the effects of diazoxide in different time points the statistical analysis between the groups was performed even when the over time assessments were not statistically significant. As appropriate, either a Student $t$ test or a Mann-Whitney U test was applied. Two tailed significance levels are reported and a $P<.05$ was considered statistically significant.

\section{RESULTS}

\section{Comparability of Study Groups}

Total of 15 piglets were operated. To ensure comparable study groups, three of the piglets were excluded. Coronary anatomy in one piglet was significantly divergent compared to

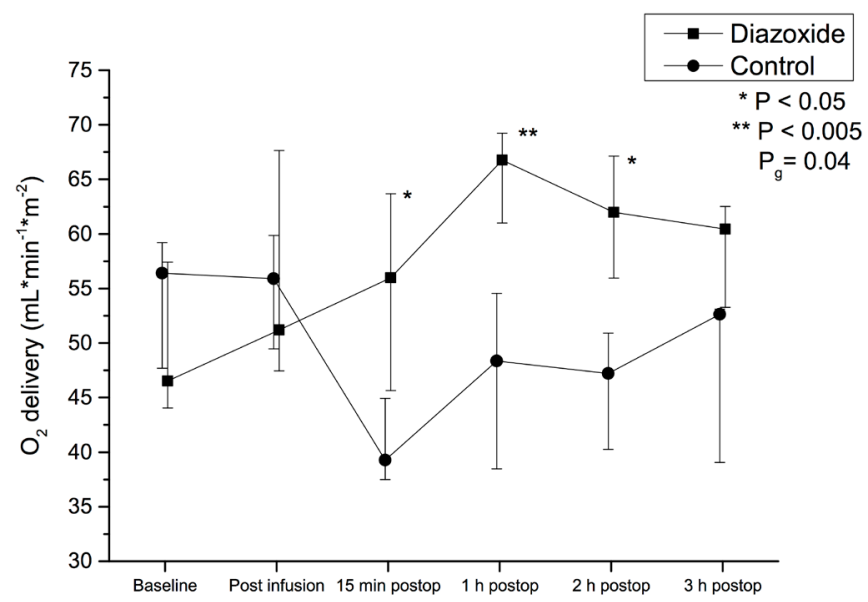

Figure 5. Oxygen delivery. After the onset of reperfusion, the delivery of oxygen was significantly higher in the diazoxide group while the baseline values were in favor of the control group. The most significant difference was seen at one hour postoperatively.

other animals as the LCX seemed to be the major contributor to coronary circulation. One piglet was excluded due to mitral regurgitation and another piglet was excluded due to a persistent ventricular fibrillation after the LCX occlusion. Eventually, six animals were included into each group. In both groups, defibrillation $(50 \mathrm{~J})$ due to ventricular tachycardia was used with one animal. TEM analyses were performed to five piglets of both groups as one cardiac sample in both groups contained only fat and connective tissue due to technical difficulties.

The mean weight of the piglets was $22.4 \mathrm{~kg}$ in the diazoxide group and $21.7 \mathrm{~kg}$ in the control group $(P=.629)$. Blood and rectal temperatures did not differ between the groups $\left(P \mathrm{t}^{*} \mathrm{~g}=.487\right.$ and $P \mathrm{t}^{*} \mathrm{~g}=.609$, respectively). There were no differences in mean arterial pressure (MAP) between the groups at any time point $\left(P^{*} \mathrm{~g}=.190\right)$. Heart rate tended to be higher in the diazoxide group, particularly at two and three hours after the reperfusion $(P=.025$ in both) but statistical significance was not reached between the groups over time $\left(P \mathrm{t}^{*} \mathrm{~g}=.061\right)$. Hemodynamic and metabolic data are summarized in Tables 2 and 3.

\section{Cardiac Function}

Creatine kinase isoenzyme MB release was significantly lower in the diazoxide group at several time points after the reperfusion of LCX (Table 3). Also, statistically significant difference $\left(P \mathrm{t}^{*} \mathrm{~g}=.030\right)$ was seen between the groups over time (Figure 1). Troponin I levels were lower in the diazoxide group (Figure 2), notably at two and three hours postoperatively $(P=.037$ in both).

Cardiac index values indicate better cardiac function in the diazoxide group with statistically significant differences at one and two hours postoperatively $(P=.016$ and $P=.037$, respectively), as seen in Figure 3.

\section{Oxygen Metabolism}

Oxygen consumption differed significantly between the groups over time with the higher values in the diazoxide group after the LCX reperfusion $\left(P \mathrm{t}^{*} \mathrm{~g}=.037\right)$ as seen in Figure 4. In 
Table 2. Hemodynamic Data

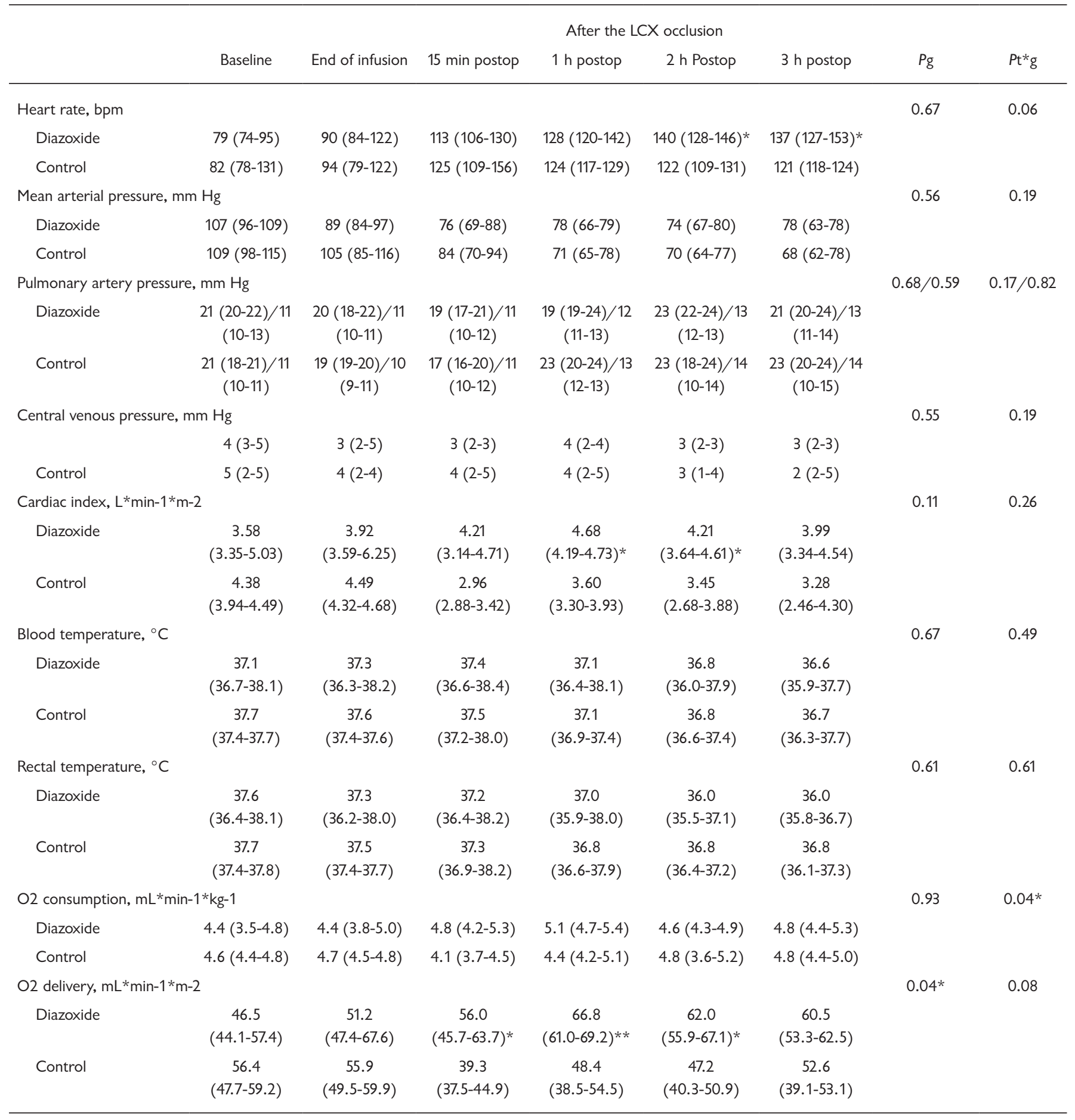

Diazoxide, $\mathrm{n}=6$; control, $\mathrm{n}=6$. Values are shown as medians and 25 th and 75 th percentiles. $* P<.05$ at single time point. $* * P<.005$ at single time point.

addition, the delivery of oxygen was observed to be higher in the diazoxide group $(P g=.038)$. The most defining difference occurred one hour postoperatively, as presented in Figure 5. Oxygen metabolic data is summarized in Table 2.

\section{Transmission Electron Microscopy Findings}

Transmission electron microscopy revealed that the total ischemic damage to myocardial ultrastructure was less in the diazoxide group than in the control group $(P=.004)$, as 
Table 3. Metabolic Data

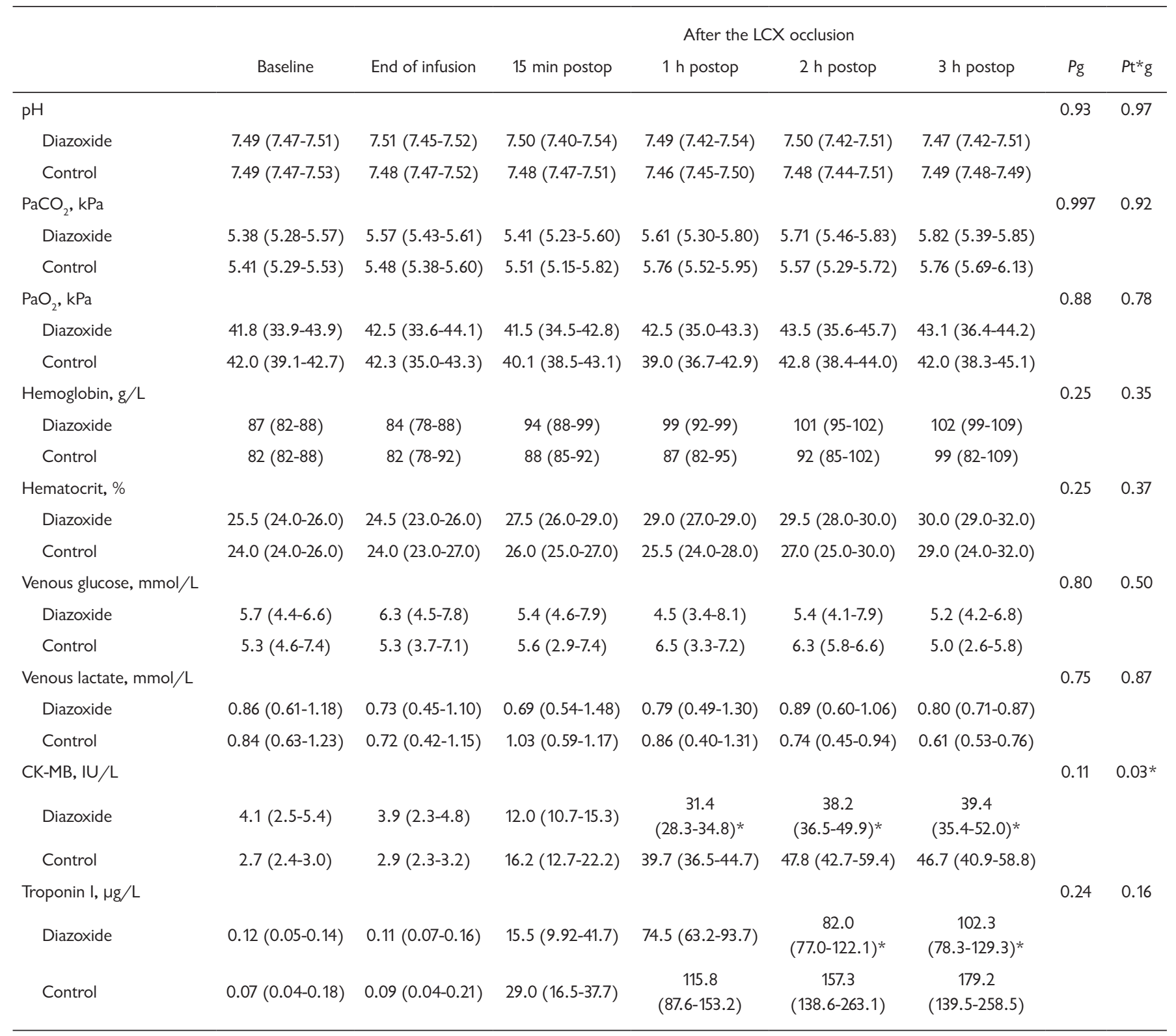

Diazoxide, $\mathrm{n}=6$; control, $\mathrm{n}=6$. Values are shown as medians and 25 th and 75 th percentiles. ${ }^{\star} P<.05$ at single time point.

seen in Table 4. Mitochondria retained their normal shape in 2 of 5 samples in the diazoxide group, whereas normal mitochondria were not seen in the samples of the control group. Furthermore, all 5 samples in the control group showed mitochondrial swelling with amorphous matrix densities and disorganized cristae (Figure 6). In the diazoxide group the combination was seen in one sample from the infarcted area $(P=.018)$.

The cardiomyocytes of the diazoxide group showed less ischemic damage $(P=.005)$. Vacuolization, loss of normal cardiomyocyte architecture and disintegrating sarcolemma were more apparent in the control group, as well as peripherally aggregated nuclear chromatin and lysis. Neutrophil aggregation was considerably milder in the diazoxide group $0.2(0.45)$ than in the control group $0.8(0.45)$, with a borderline significant difference $(P=.067)$. Interestingly, 4 of the samples in the control group showed intramyocytic fat deposition (IMFD), when that in the diazoxide group was in 2 samples, of which one had only mild IMFD $(P=.221)$.

\section{Histopathological and Immunobistochemical Analysis}

The immunohistochemical staining score of OGG1 was significantly greater in the control group in the left anterior ventricle $(P=.021)$. The total scores of Nrf2, 8-OHdG and 
Table 4. Transmission Electron Microscopy Findings

\begin{tabular}{lccc}
\hline Protocol & Mitochondria & Cardiomyocytes & Total Score \\
\hline Diazoxide & $0.8(0.84)$ & $0.2(0.45)$ & $1.0(1.23)$ \\
Control & $2.0(0.00)$ & $1.4(0.55)$ & $3.4(0.55)$ \\
$P$ & $.018^{*}$ & $.005^{*}$ & $.004^{* *}$ \\
\hline
\end{tabular}

Diazoxide, $\mathrm{n}=6$; control, $\mathrm{n}=6$. Values are shown as means and standard deviations. $* P<.05 . * * P<.005$.

PKC $\varepsilon$ did not differ statistical significantly between the study groups $(P=.805, P=.686, P=.492$, respectively). Immunohistochemical findings are summarized in Table 5.

The mean sum of histopathological total scores was 6.8 (1.47) in the diazoxide group and $8.0(2.10)$ in the control group $(P=.291)$. The main finding was eosinophilia $(P=.083)$, with the greater values in the control group. Capillary congestion $(P=.780)$ and granulocyte infiltration $(P=.651)$ did not show differences between the groups.

\section{DIsCussion}

Pharmacological preconditioning has been studied for benefits in reducing ischemic myocardial injury. Fryer and colleagues suggest that mitoK $\mathrm{K}_{\text {ATP }}$ are the key targets of preconditioning [Fryer 2001]. In this study, we show that mito$\mathrm{K}_{\mathrm{ATP}}$-channel opener diazoxide administered prior to ischemia alleviates the myocardial ischemic damage.

On a cellular level the diazoxide group fared better. The transmission electron microscopy showed that cell structure was better preserved and mitochondrial damage attenuated in the diazoxide group, indicating less ischemic damage. The control group showed more mitochondrial swelling and
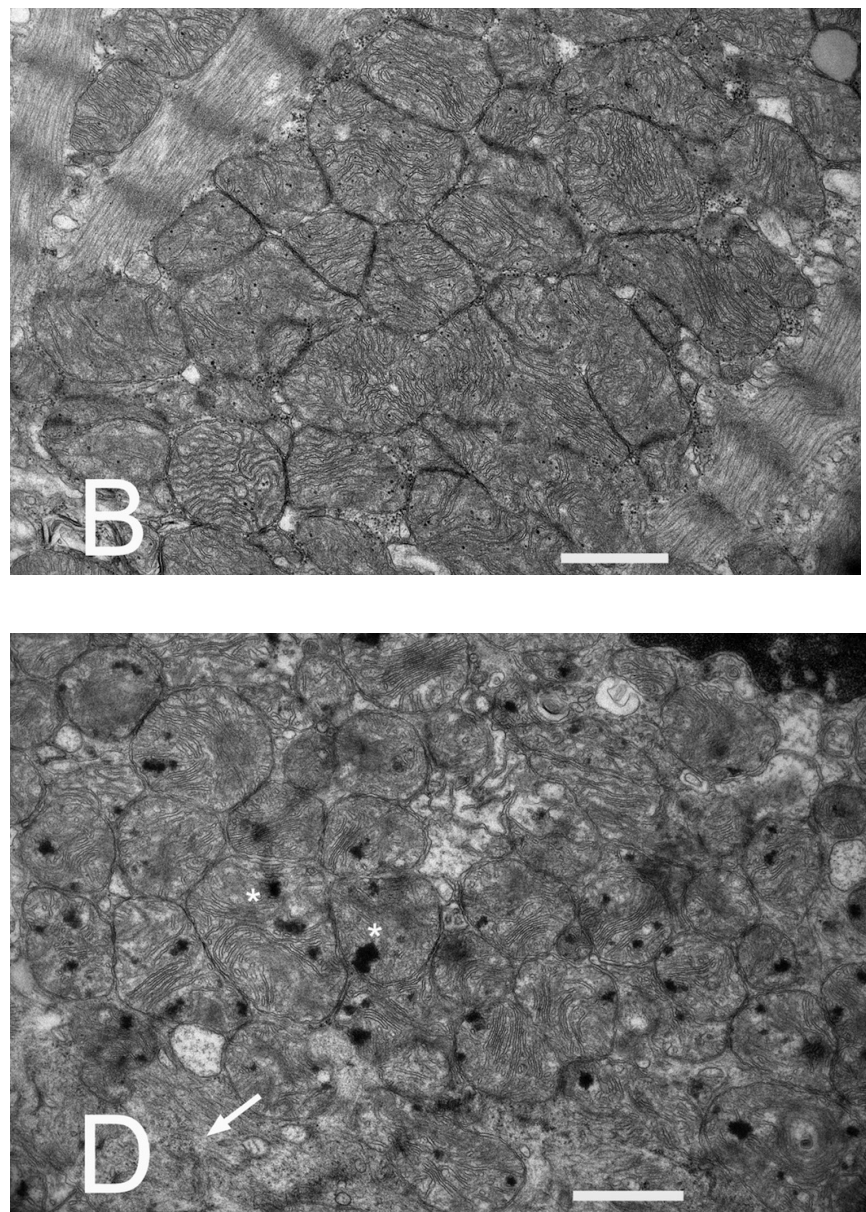

Figure 6. Representative transmission electron micrographs of infarct region in left ventricle with preoperative solution of diazoxide (A-B) or without diazoxide (C-D). A, Slight edema can be seen but myocytes have normal fibrillar architecture $(\times 2900)$. B, Slightly swollen mitochondria and mildly affected christae but no AMDs are seen $(\times 6800)$. C, Myocytes and contraction bands are affected (arrows). Mitochondria are swollen and AMDs are apparent (*), magnification $\times 2900$. D, Mitochondria are swollen and some are losing their normal architecture. Fibrillar architecture has also disappeared (arrow). Disorganized cristae as well as AMDs are seen $(*)$. Magnifcation $\times 6800$. Scale bars: $\mathbf{2} \boldsymbol{\mu m}(\mathbf{A}, \mathbf{C})$ and I $\boldsymbol{\mu m}(\mathbf{B}, \mathbf{D})$. 
Table 5. Immunohistochemistry

\begin{tabular}{|c|c|c|c|c|c|c|c|}
\hline \multicolumn{8}{|l|}{$\mathrm{Nrf2}$} \\
\hline & Control & $3.0(0.00)$ & $1.3(1.03)$ & $2.0(0.89)$ & $1.2(0.75)$ & $2.7(0.52)$ & $10.2(2.64)$ \\
\hline & $P$ & .317 & .849 & .484 & .665 & 0.465 & 0.805 \\
\hline & Diazoxide & $1.8(1.17)$ & $0.5(0.55)$ & $0.7(1.21)$ & $0.5(0.84)$ & $1.7(1.37)$ & $5.2(2.71)$ \\
\hline & Control & $1.2(0.75)$ & $0.5(0.84)$ & $1.2(1.17)$ & $0.3(0.52)$ & $1.8(1.17)$ & $5.0(2.83)$ \\
\hline & $P$ & .243 & .784 & .342 & .847 & 0.867 & 0.686 \\
\hline \multicolumn{8}{|l|}{ OGG1 } \\
\hline & Diazoxide & $2.5(0.55)$ & $2.2(0.75)$ & $2.7(0.82)$ & $2.7(0.52)$ & $2.7(0.52)$ & $12.7(1.75)$ \\
\hline & Diazoxide & $2.2(0.41)$ & $1.3(0.52)$ & $1.8(0.41)$ & $1.0(0.00)$ & $2.2(0.41)$ & $8.5(0.84)$ \\
\hline & Control & $2.0(0.63)$ & $1.5(0.55)$ & $1.7(0.52)$ & $1.0(0.00)$ & $2.0(0.63)$ & $8.2(1.17)$ \\
\hline & $P$ & .598 & .575 & .523 & $>.999$ & 0.598 & 0.492 \\
\hline
\end{tabular}

Diazoxide, $\mathrm{n}=6$; control, $\mathrm{n}=6$. Values are shown as means and standard deviations. $* P<.05$.

disorganized cristae with amorphous matrix densities, which were not seen in the diazoxide group. These mitochondrial densities may be due to an increased accumulation of mitochondrial $\mathrm{Ca}^{2+}$, as it is shown that opening of mitoK ${ }_{\text {ATP }}$-channels attenuates mitochondrial $\mathrm{Ca}^{2+}$ overload [Holmuhamedov 1999; Murata 2001]. Diazoxide diminishing mitochondrial densities, swelling and cristae damage were also recorded in a study modeling acute myocardial infarction, where diazoxide was added to cardioplegia [McCully 2002].

Supporting findings of the cardioprotective effect of mito$\mathrm{K}_{\mathrm{ATP}}$ are the lower levels of cardiac markers CK-MBm and Troponin I in the diazoxide group. Deja and Shahid have previously reported similar findings [Deja 2006; Shahid 2008]. Interestingly, we also observed different oxygen metabolism profiles between the groups. Higher rate of oxygen consumption and delivery was found in the diazoxide group during reperfusion phase, which suggests that cellular function may be better. This would make sense keeping in mind the effect of mitoKATP being open during reperfusion (ATP hydrolysis reaction). Also, the cardiac index for the diazoxide group was significantly better at 1 and 2 hours postoperatively.

The oxidative stress analysis could not differentiate the groups, except for OGG1 in one area of the heart. The histopathological scores tended to favor the diazoxide group, but this did not reach statistically significant difference. The lack of immunohistochemical and histopathological findings might be due to the fact of a short follow-up period or that the ischemia inflicted was not severe enough to see a difference. It is also notable that various previous studies have used quite a different scale of infused diazoxide, varying between 1-10 milligrams $/ \mathrm{kg}$, whereas in our study we used $3.5 \mathrm{mg} / \mathrm{kg}$. Higher dosages have proven to be toxic, but dosages that are too low have proven to be ineffective [Kowaltowski 2001]. It can be speculated that if our dosage had been higher, we might have seen a more clear difference in the oxidative stress markers.

Coetzee summarizes the cardioprotective effects of diazoxide to include reduction in infarct size, less CK leakage, and better post infarct functionality [Coetzee 2013]. The main finding in our present study is less damage to myocardial cellular structure in the diazoxide group. Our findings are in line with previous findings.

To conclude, our study suggests that diazoxide is beneficial in preserving myocardial cellular structure and cellular function. Better survived mitochondria can use more oxygen as higher oxygen delivery and consumption indicated. Furthermore this enables higher cardiac function. Diazoxide may have benefits in treating ischemic heart injury.

\section{ACKNOWLEDGEMENT}

The authors would like to thank Pasi Ohtonen, MSc, for his assistance in statistical analysis and Seija Seljänperä, RN, for her help in caring for the animals.

\section{REFERENCES}

Baines CP, Liu GS, Birincioglu M, Critz SD, Cohen MV, Downey JM. 1999. Ischemic preconditioning depends on interaction between mitochondrial KATP channels and actin cytoskeleton. Am J Physiol 276(4 Pt 2):H1361-8. 
Baines CP, Song C, Zheng Y, et al. 2003. Protein kinase ce interacts with and inhibits the permeability transition pore in cardiac mitochondria. Circ Res 92:873-80.

Coetzee WA. 2013. Multiplicity of effectors of the cardioprotective agent, diazoxide. Pharmacol Ther 140:167-75.

de Souza-Pinto NC, Eide L, Hogue BA, et al. 2001. Repair of 8-oxodeoxyguanosine lesions in mitochondrial dna depends on the oxoguanine dna glycosylase (OGG1) gene and 8-oxoguanine accumulates in the mitochondrial dna of OGG1-defective mice. Cancer Res 61:5378-81.

Deja MA, Golba KS, Malinowski M, et al. 2006. Diazoxide provides maximal KATP channels independent protection if present throughout hypoxia. Ann Thorac Surg 81:1408-16.

Deja MA, Malinowski M, Golba KS, et al. 2009. Diazoxide protects myocardial mitochondria, metabolism, and function during cardiac surgery: A double-blind randomized feasibility study of diazoxide-supplemented cardioplegia. J Thorac Cardiovasc Surg 137:997-1004.e2.

Fryer RM, Hsu AK, Gross GJ. 2001. Mitochondrial K(ATP) channel opening is important during index ischemia and following myocardial reperfusion in ischemic preconditioned rat hearts. J Mol Cell Cardiol $33: 831-4$.

Fryer RM, Eells JT, Hsu AK, Henry MM, Gross GJ. 2000. Ischemic preconditioning in rats: Role of mitochondrial KATP channel in preservation of mitochondrial function. Am J Physiol Heart Circ Physiol 278:H305-12.

Garlid AO, Jaburek M, Jacobs JP, Garlid KD. 2013. Mitochondrial reactive oxygen species: Which ROS signals cardioprotection? Am J Physiol Heart Circ Physiol 305:H960-8.

Garlid KD, Paucek P, Yarov-Yarovoy V, et al. 1997. Cardioprotective effect of diazoxide and its interaction with mitochondrial ATP-sensitive $\mathrm{K}+$ channels: Possible mechanism of cardioprotection. Circ Res 81:1072-82.

Ghosh S, Standen NB, Galinanes M. 2000. Evidence for mitochondrial $\mathrm{K}$ ATP channels as effectors of human myocardial preconditioning. Cardiovasc Res 45:934-40.

Griffiths EJ, Halestrap AP. 1995. Mitochondrial non-specific pores remain closed during cardiac ischaemia, but open upon reperfusion. Biochem J 307:Pt 1/Pt 1:93-8.

Holmuhamedov EL, Wang L, Terzic A. 1999. ATP-sensitive K+ channel openers prevent $\mathrm{Ca} 2+$ overload in rat cardiac mitochondria. J Physiol 519:Pt 2:347-60.

Itoh K, Chiba T, Takahashi S, et al. 1997. An Nrf2/small maf heterodimer mediates the induction of phase II detoxifying enzyme genes through antioxidant response elements. Biochem Biophys Res Commun 236:313-22.

Itoh K, Wakabayashi N, Katoh Y, et al. 1999. Keap1 represses nuclear activation of antioxidant responsive elements by Nrf2 through binding to the amino-terminal Neh2 domain. Genes Dev 13:76-86.

Jensen HA, Loukogeorgakis S, Yannopoulos F, et al. 2011. Remote ischemic preconditioning protects the brain against injury after hypothermic circulatory ArrestClinical perspective. Circulation 123:714-21.

Kowaltowski AJ, Seetharaman S, Paucek P, Garlid KD. 2001. Bioenergetic consequences of opening the ATP-sensitive $\mathrm{K}(+)$ channel of heart mitochondria. Am J Physiol Heart Circ Physiol 280:H649-57.

Li H, Yang T, Long Z, Cheng J. 2014. Effect of mitochondrial ATP-sensitive potassium channel opening on the translocation of protein kinase $\mathrm{C}$ epsilon in adult rat ventricular myocytes. Genet Mol Res 13:4516-22.

McCully JD, Wakiyama H, Cowan DB, Federman M, Parker RA, Levitsky S. 2002. Diazoxide amelioration of myocardial injury and mitochondrial damage during cardiac surgery. Ann Thorac Surg 74:2138-45; discussion 2146

Mizutani S, Al-Dadah AS, Bloch JB, et al. 2006. Hyperkalemic cardioplegia-induced myocyte swelling and contractile dysfunction: Prevention by diazoxide. Ann Thorac Surg 81:154-9.

Murata M, Akao M, O’Rourke B, Marban E. 2001. Mitochondrial ATPsensitive potassium channels attenuate matrix $\mathrm{ca}(2+)$ overload during simulated ischemia and reperfusion: Possible mechanism of cardioprotection. Circ Res 89:891-8.

Murry CE, Jennings RB, Reimer KA. 1986. Preconditioning with ischemia: A delay of lethal cell injury in ischemic myocardium. Circulation 74:1124-36.

Sanada S, Kitakaze M, Asanuma H, et al. 2001. Role of mitochondrial and sarcolemmal KATP channels in ischemic preconditioning of the canine heart. Am J Physiol Heart Circ Physiol 280:H256-63.

Sellitto AD, Maffit SK, Al-Dadah AS, et al. 2010. Diazoxide maintenance of myocyte volume and contractility during stress: Evidence for a non-sarcolemmal KATP channel location. J Thorac Cardiovasc Surg 140:1153-9.

Shahid M, Tauseef M, Sharma KK, Fahim M. 2008. Brief femoral artery ischaemia provides protection against myocardial ischaemia-reperfusion injury in rats: The possible mechanisms. Exp Physiol 93:954-68.

Tsutsui H, Ide T, Kinugawa S. 2006. Mitochondrial oxidative stress, DNA damage, and heart failure. Antioxid Redox Signal 8:1737-44.

Valavanidis A, Vlachogianni T, Fiotakis C. 2009. 8-hydroxy-2' -deoxyguanosine (8-OHdG): A critical biomarker of oxidative stress and carcinogenesis. J Environ Sci Health C Environ Carcinog Ecotoxicol Rev 27:120-39.

Yang JC, Cortopassi GA. 1998. Induction of the mitochondrial permeability transition causes release of the apoptogenic factor cytochrome $\mathrm{C}$ Free Radic Biol Med 24:624-31. 